\title{
Achievable Closed-Loop Properties of Systems Under Decentralized Control: Conditions Involving the Steady-State Gain
}

\author{
Peter J. Campo and Manfred Morari, Member, IEEE
}

\begin{abstract}
The question of the existence of decentralized controllers for open-loop stable multivariable systems which provide particular closed-loop properties is investigated. In particular, we study the existence of decentralized controllers which provide integral action (Type 1 closed-loop performance) and also demonstrate one or more of: unconditional stability, integrity with respect to actuator and sensor failure, and decentralized unconditional stability. Necessary, sufficient, and, in some cases, necessary and sufficient conditions on the open-loop steady-state gain are derived such that there exists a controller which provides these desired closed-loop characteristics. These results provide the basis for a systematic approach to control structure selection for decentralized controller design.
\end{abstract}

\section{INTRODUCTION}

D ESPITE the closed-loop performance advantages of multivariable controllers, the use of single loop controllers for multivariable plants is the rule in industrial process control applications. In addition to its inherent simplicity, a decentralized control system consisting of independent controller subsystems (typically single input-single output (SISO) control loops) exhibits several advantages over a fully multivariable design. In the ideal case these advantages include:

1) Flexibility in Operation. A decentralized structure allows operating personnel to restructure the control system by bringing subsystems in and out of service individually. This flexibility allows the system to handle changing control objectives during different operating conditions (for example start-up, shutdown, temporary process modifications to accommodate maintenance, etc.).

2) Failure Tolerance. The flexibility characteristics of the decentralized structure also provide advantages in the case of unanticipated structural changes in the control system. In particular, if an actuator or sensor fails, only the individual subsystem involved is affected. Only this subsystem need be taken out of service with no changes to other parts of the control system.

3) Simplified Design. Sequential design of individual SISO subsystems is usually easier than a full multivariable design. In particular, the number of design parameters

Manuscript received March 26, 1992; revised May 10, 1993. Recommended by Past Associate Editor, J. S. Freudenberg.

P. J. Campo is with General Electric Corporate Research and Development, Schenectady, NY 12301 USA.

M. Morari is with the Department of Chemical Engineering, California Institute of Technology, Pasadena, CA 91125 USA

IEEE Log Number 9216624. which must be specified in each SISO design is typically much smaller than a full multivariable design.

4) Simplified Tuning. Individual subsystems can be (manually) tuned and retuned on-line to accommodate the effects of (slowly) changing process conditions.

The requirement that the control system be decentralized introduces the pairing problem. The pairing problem is concerned with defining the control system structure, i.e., which of the available plant inputs is to be used to control each of the plant outputs. For a fully noninteracting plant, the choice is obvious, and the benefits of decentralized control discussed above accrue trivially. In any practical problem, there are (to a greater or lesser extent) interactions in the plant. This implies that even if the control system is decentralized, subsystems of the closed-loop system are not independent of each other. To the extent that the control system can be designed to make the closed-loop subsystems independent, the idealized characteristics outlined above can be realized.

When the process interactions are significant, the choice of a control system structure is far from trivial and has been the subject of much research [17], [24], [25], [28], [33], [35]. For an $n \times n$ plant there are $n$ ! possible SISO loop pairings. For plants beyond even a modest number of inputs and outputs, a brute force approach (to design controllers for every possible pairing and then select the design which provides the best closed-loop performance) is impractical. ${ }^{1}$

This complexity drives the need for analysis methods to determine achievable closed-loop system characteristics as a function of control system structure independent of controller design. With these tools, pairings which do not admit acceptable closed-loop performance can be discarded before any controllers are designed. The development of such tools and their use in the pairing problem is the subject of this paper. An important characteristic of the results developed here is that they only require steady-state knowledge of the plant. This information is easily obtained from simple identification experiments or steady-state design models. Often the pairing question can be answered without the need for detailed dynamic modeling which, in itself, can be expensive and time consuming for large scale systems.

${ }^{1}$ Even if design and performance evaluation could be completed in one second, this process would require 1000 hours to complete for a plant with 10 inputs and 10 outputs. 
Several authors have reported on work in this area. Grosdidier and Morari [16] took a similar approach to the study of single loop controllers for multivariable plants. They defined the concepts of integral stabilizability and integral controllability which are generalized here. Skogestad and Morari [33] introduce the important notion of decentralized integral controllability (DIC) and give some necessary and some sufficient conditions for a system to be DIC. The work of Morari and coworkers is nicely summarized in [27]. Chiu and Arkun [7] study the problem of failure tolerance in a similar setting. In this paper, we bring these ideas together in a unified way and present a number of novel results which generalize those available to date.

\section{Preliminary Definitions}

The general system under study is shown in Fig. 1. We make the following assumptions throughout the paper.

1) The $n$ input $n$ output plant, $G(s)$, is finite dimensional, linear time invariant, stable, and strictly proper.

2) Type 1 closed-loop behavior (i.e., integral action) is required for all $n$ plant outputs.

3) The controller, $K(s)$, is finite dimensional, linear time invariant, proper, and of the form

$$
K(s)=\frac{1}{s} C(s)
$$

where $C(s)$ is diagonal, stable, and such that $C(0)$ is nonsingular, i.e., $K(s)$ is fully decentralized.

The limitation to open-loop stable plants is not restrictive since we will be interested in studying the behavior of the closed-loop system as subsystem controllers are (arbitrarily) taken out of service; in this setting it is not meaningful to consider open-loop unstable plants. The requirement of integral action is typical in process control applications and allows us to connect closed-loop system properties to the open-loop steady state gain. Given assumptions 2) and 3), the inclusion of an explicit integrator in each channel of $K(s)$ as in (1) is without loss of generality (note that $C(0)$ nonsingular is necessary to have integral action on all outputs). Most of the results to be presented can be extended to block decentralized controllers, i.e., those for which $C(s)$ is block diagonal, using concepts such as the block relative gain [24] and block $D$-stability [1], [22], although this is not pursued here. The requirement that $C(s)$ be stable imposes a certain loss of generality but this is not significant in most process control applications.

Because the controller $K(s)$ is assumed to be diagonal, the control system input-output pairings are determined by the definition of $G(s)$. To consider all possible input-output pairings we must consider all transfer function matrices, $\hat{G}(s)$, given by

$$
\hat{G}(s)=P G(s)
$$

where $P$ is a $n \times n$ permutation matrix. ${ }^{2}$

\footnotetext{
${ }^{2}$ Recall that each column of a permutation matrix consists of 1 one and $n-1$ zeros. Furthermore, permutation matrices are orthogonal so that $P^{-1}=P^{T}$.
}

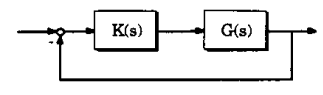

Fig. 1. The closed-loop system under study.

Given this introduction we are prepared to introduce a classification of closed-loop systems along the lines of the flexibility and on-line tuning properties discussed in Section I.

Definition 1: The system in Fig. 1 is internally stable if bounded signals injected at any point in the system give rise to bounded signals at all other points in the system.

We will use the terminology " $K(s)$ stabilizes $G(s)$," to mean that the given controller and plant form an internally stable closed-loop system when connected as in Fig. 1.

Definition 2: The closed-loop system in Fig. 1 is unconditionally stable if $\hat{K}(s)=E K(s)$ stabilizes $G(s)$ for all $E \in \mathcal{E}_{I}$ where

$$
\mathcal{E}_{I} \triangleq\{E=\alpha I \mid \alpha \in(0,1]\} .
$$

Unconditionally stable closed-loop systems allow a minimum measure of on-line tuning. In particular, the gains of each of the subsystem controllers can be simultaneously varied by a factor in the range $(0,1]$ and the system will remain stable. Unconditional stability can also be regarded as a measure of closed-loop robustness since for a particular controller, $K(s)$, unconditional stability implies that stability is maintained if the elements of $G(s)$ vary by a scalar factor as well.

Definition 3: The closed-loop system in Fig. 1 demonstrates integrity if $\hat{K}(s)=E K(s)$ stabilizes $G(s)$ for all $E \in \mathcal{E}_{1 / 0}$ where

$$
\mathcal{E}_{1 / 0} \triangleq\left\{E=\operatorname{diag}\left(\epsilon_{i}\right) \mid \epsilon_{i} \in\{0,1\}, i=1, \cdots, n\right\} .
$$

This definition of integrity implies that the closed-loop system remains stable as subsystem controllers are arbitrarily brought in and out of service (see, for example, [31]). A related definition of closed-loop integrity [15] requires the system to remain stable in the face of arbitrary sensor or actuator failures. ${ }^{3}$ Integrity in this sense requires that both $K(s)$ and $G(s)$ be stable, which precludes Type 1 closed-loop behavior and is, therefore, too restrictive for our purposes. On the other hand, integrity in the sense of Definition 3 does not imply sensor or actuator failure tolerance unless the failure is recognized and the affected control loop taken out of service.

Definition 4: The closed-loop system in Fig. 1 is decentralized unconditionally stable if $\hat{K}(s)=E K(s)$ stabilizes $G(s)$ for all $E \in \mathcal{E}_{D}$ where

$$
\mathcal{E}_{D} \triangleq\left\{E=\operatorname{diag}\left(\epsilon_{i}\right) \mid \epsilon_{i} \in[0,1], i=1, \cdots, n\right\} .
$$

A closed-loop system which is decentralized unconditionally stable allows the gains of each controller subsystem to be modified independently by a factor in the range $[0,1]$. Note that the gains can be made zero which corresponds to taking a subsystem out of service. Thus decentralized unconditional stability implies closed-loop integrity.

${ }^{3}$ With $K(s)$ diagonal, integrity with respect to sensor failures is equivalent to integrity with respect to actuator failures. 
While these definitions are useful for classifying closedloop systems, they are of no direct use in the pairing problem because they require the specification of a controller, $K(s)$, a priori. To overcome this limitation, we define a parallel classification of open-loop systems. In particular, we classify a given open-loop system, $G(s)$, according to whether or not there exists a controller of the form (1) which together with $G(s)$ demonstrates a particular closed-loop property. Formally we define:

Definition 5: The plant $G(s)$ is integral stabilizable (IS) if there exists a controller, $K(s)$, of the form (1) which stabilizes $G(s)$.

Definition 6: The plant $G(s)$ is integral controllable (IC) if there exists a controller, $K(s)$, of the form (1) such that the system in Fig. 1 is unconditionally stable.

Definition 7: The plant $G(s)$ is integral controllable with integrity (ICI) if there exists a controller, $K(s)$, of the form (1) such that the system in Fig. 1 demonstrates integrity.

Definition 8: The plant $G(s)$ is decentralized integral controllable (DIC) if there exists a controller, $K(s)$, of the form (1) such that the system in Fig. 1 is decentralized unconditionally stable.

Since only existence of a suitable controller is required in Definitions 5-8, the dependence on $K(s)$ inherent in Definitions $1-4$ is removed. In Section IV we develop analysis methods which allow us to classify a given $G(s)$ according to Definitions 5-8 without having to explicitly construct $K(s)$. Since the classifications defined by Definitions $5-8$ depend on the choice of controller structure through the definition of $G(s)$, these analysis methods allow us to study the suitability of various potential control structures without having to complete detailed controller designs. In particular we can determine if given closed-loop flexibility and on-line tuning characteristics are achievable for a particular plant and controller structure independent of controller design.

Morari [26], Grosdidier and Morari [16], and Morari and Zafiriou [27] present more limited definitions of integral stabilizability and integral controllability. Their definitions involve properties of a closed-loop system with a given $K(s)$ and correspond to the definitions of internal stability and unconditional stability presented here. Our motivation in generalizing these definitions is to make them independent of $K(s)$ and consistent with the definition of decentralized integral controllability originally introduced by Skogestad and Morari [33].

The remainder of the paper is devoted to developing conditions on $G(s)$ which allow us to classify it according to Definitions 5-8. These results take the form of necessary, sufficient, or, where possible, necessary and sufficient conditions for membership in a particular class. Because a number of conflicting definitions and results have appeared in the literature, we include results for each of IS, IC, ICI, and DIC. Our main focus, however, is on the richer system properties ICI and DIC. The primary reasons for this are the engineering significance and relative absence of existing results for these classes.

To make the presentation clearer and more self contained, known results are interspersed with new results as appropriate.
A theorem-proof approach is adopted for rigor, with numerous remarks following each result explaining its significance and offering illuminating examples. All new results are proven in detail; proofs of known results are provided only when they are not readily available elsewhere or when the proof adds clarity to the following exposition. Notation is introduced as required and summarized in Appendix A.

\section{CloSED-LOOP SYSTEM PROPERTIES}

We begin with a brief characterization of the closed-loop classifications of Definitions 1-4. These results are not meant to be definitive-more sophisticated closed loop analysis tools, such as the real multivariable gain margin or the structured singular value, could be used to study the closed-loop problem. Our purpose here, however, is only to state those results which are required to for the more interesting open-loop classifications in Section IV.

\section{A. Internal Stability}

The following well-known result provides a necessary and sufficient condition for internal stability of the system in Fig. 1.

Fact 1: Given that $G(s)$ is stable, the closed-loop system of Fig. 1 is internally stable if and only if $Q(s) \triangleq K(s)[I+$ $G(s) K(s)]^{-1}$ is stable.

This result is a special case of the Youla parameterization of all stabilizing controllers (see for example [14]). For a discussion and proof with the notation used here see [27]

\section{B. Unconditional Stability}

Using $\sigma[\cdot]$ to denote the spectrum of a matrix argument and $\overline{\mathcal{C}}_{+}-\{0\}$ to denote the closed right-half plane less the origin, the following holds from Theorem 3 of [26].

Fact 2: The system of Fig. 1 is unconditionally stable only if $\sigma[G(0) C(0)] \subset \overline{\mathcal{C}}_{+}-\{0\}$.

Fact 3: Given $C(s)$ diagonal and stable, there exists $\gamma>0$ sufficiently small such that the system of Fig. 1 with $K(s)=$ ${ }_{s}^{\gamma} C(s)$ is unconditionally stable if $\sigma[G(0) C(0)] \subset \mathcal{C}_{+}$.

Except in the case that $G(0) C(0)$ has purely imaginary eigenvalues, Facts 2 and 3 completely characterize unconditionally stable closed-loop systems. Example 4 of Grosdidier and Morari [16] demonstrates that nothing can be said about unconditional stability from $G(0) C(0)$ alone when its spectrum contains purely imaginary values.

\section{Integrity}

To study the situation where parts of the controller can be taken out of service, we need the notions of principal submatrices and principal subsystems.

The index set $M$ consisting of $k$ tuples of integers in the range $1, \cdots, n$ is defined by

$$
M \triangleq\left\{\left(i_{1}, \cdots, i_{k}\right) \mid 1 \leq i_{1}<i_{2} \cdots<i_{k} \leq n\right\} .
$$

Given an $n \times n$ matrix $A$, each $m=\left\{i_{1}, \cdots, i_{k}\right\} \in M$ defines a $k \times k$ principal submatrix of $A$, denoted $A_{m}$, made up of the rows and columns of $A$ indexed by $m$. For a given $m \in M$, the notation, $\operatorname{dim}(m)$, refers to the number of elements in $m$ 
and indicates the dimension of the principal submatrix, $A_{m}$. Given a system represented by the transfer function matrix $G(s)$, its principal subsystems are represented by the transfer function matrices $G_{m}(s)$, the principal submatrices of $G(s)$.

Given this notation the following is a direct consequence of Definition 3.

Fact 4: The closed-loop system of Fig. 1 demonstrates integrity if and only if $K_{m}(s)$ stabilizes $G_{m}(s)$ for all $m \in M$.

\section{Decentralized Unconditional Stability}

The following simple result follows from Definitions 2-4.

Fact 5: The closed-loop system of Fig. 1 is decentralized unconditionally stable if and only if for all diagonal $D>0$ and all $m \in M,\left(1 /\|D\|_{2}\right)[D K(s)]_{m}$ unconditionally stabilizes $G_{m}(s){ }^{4}$

Trivially, decentralized unconditional stability implies that $K(s)$ stabilizes $G(s)$ with integrity and that $K_{m}(s)$ unconditionally stabilizes $G_{m}(s)$. This follows directly from Fact 5 in the special case $D=I$.

\section{OPEN-LOOP SySTEM PROPERTIES}

We now turn our attention to the characterization of openloop systems. These results allow us to answer the question, "For a given plant and variable pairing defined by $G(s)$, does there exist a controller of the form (1) which together with $G(s)$ forms a closed-loop system with property $X$ ?" where property $X$ is any one or more of: internal stability, unconditional stability, integrity, or decentralized unconditional stability. Since the answer depends upon the variable pairing chosen, these results provide us with quantitative measures for evaluating the suitability of potential control system structures without having to develop controller designs.

\section{A. Integral Stabilizability}

A complete characterization of the IS property is provided by the following theorem involving an easily verified condition.

Theorem 1: $G(s)$ is integral stabilizable if and only if $G(0)$ is nonsingular.

Proof: This result is basically contained in Theorem 3 of Davison [8]. Necessity is easily demonstrated using Fact 1 above. Sufficiency follows from the observation that the augmented plant, $(1 / s) G(s)$, can have no unstable decentralized fixed modes.

Remarks:

1) The condition that $G(0)$ be nonsingular, and hence the IS property, is pairing independent. In fact, the necessity of a nonsingular steady state gain matrix can be established for any controller of the form (1), even if $C(s)$ is a full transfer function matrix, i.e., even if the controller is not decentralized.

2) The condition that $G(0)$ be nonsingular is equivalent to the requirement that the plant have no transmission zeros at the origin.

${ }^{4}$ Throughout the paper we use $\|\cdot\|_{p}$ to denote the matrix norm induced by the Holder $p$-norm on $\mathcal{R}^{n}$.

\section{B. Integral Controllability}

The following necessary condition follows from Fact 2 .

Theorem 2: $G(s)$ is integral controllable only if there exists a diagonal matrix, $X$, such that $\sigma[G(0) X] \subset \mathcal{C}_{+}-\{0\}$.

Proof: We establish the result by contradiction. Assume that no such $X$ exists but that $G(s)$ is IC. This implies that there exists a $K(s)$ of the form (1) with $C(0)$ nonsingular such that $K(s)$ unconditionally stabilizes $G(s)$. By Fact 2 this implies that $\sigma[G(0) C(0)] \subset \overline{\mathcal{C}}_{+}-\{0\}$, but this contradicts the assumption.

In a similar fashion we can use Fact 3 to develop a sufficient condition on $G(0)$ such that $G(s)$ is IC.

Theorem 3: If there exists a diagonal matrix, $X$, such that $\sigma[G(0) X] \subset \mathcal{C}_{+}$, then $G(s)$ is IC.

Proof: By Fact 3, under the given conditions there exists a $\gamma>0$ such that the controller $K(s)=(\gamma / s) X$ unconditionally stabilizes $G(s)$. The existence of such a $K(s)$ implies that $G(s)$ is IC by definition.

Remarks:

1) The condition of Theorem 3 implies not only that $G(s)$ is IC, but also that there exists a "pure integral" controller of the form, $K(s)=(\gamma / s) X$, with $X$ a constant matrix, which demonstrates that $G(s)$ is IC i.e., which unconditionally stabilizes $G(s)$.

2) The reverse direction of Theorem 3 is known not to hold. (Example 4 of [16] provides a counterexample.)

Any condition on $G(0)$ which implies that there exists an $X$ satisfying the condition of Theorem 3 implies that $G(s)$ is IC. The least restrictive such result known is provided by the following lemma.

Lemma 1: Given any matrix, $A$, if there exists a permutation matrix, $P$, such that the leading principal submatrices of $P A P^{T}$ are nonsingular, then there exists a diagonal matrix, $X$, such that $\sigma[A X] \subset \mathcal{C}_{+}$. Furthermore, if the determinants of the leading principal submatrices of $P A P^{T}$ are positive, $X$ may be chosen to be positive definite.

Proof: That there exists such an $X$ was proven originally by Fisher and Fuller [13]. An alternative proof due to Ballantine [3] demonstrates that when the determinants of the leading principal submatrices are positive, $X$ may be chosen to be positive definite.

Using Lemma 1, the following corollary follows directly from Theorem 3 .

Corollary 1: If there exists a permutation matrix, $P$, such that the leading principal submatrices of $P G(0) P^{T}$ are nonsingular, then $G(s)$ is IC.

Proof: By Lemma 1, under the given conditions there exists a diagonal $X$ such that $\sigma[G(0) X] \subset \mathcal{C}_{+}$. By Theorem 3 this implies that $G(s)$ is IC.

Remarks:

1) Corollary 1 may be equivalently restated as: If there exists a sequence of nonsingular principal submatrices, $G_{m_{1}}(0), G_{m_{2}}(0), \cdots, G_{m_{n}}(0)$, with $G_{m_{k}}(0)$ of order $k$ and $m_{i} \subset m_{j} \forall i<j$, then $G(s)$ is IC.

2) Theorems 2.2 and 2.3 of Guardabassi et al., [18] provide an equivalent result with a more involved proof. 
Except in the case that there exists a diagonal matrix $X$ which provides $\sigma[G(0) X] \subset \overline{\mathcal{C}}_{+}-\{0\}$, but no such $X$ which provides $\sigma[G(0) X] \subset \mathcal{C}_{+}$, Theorems 2 and 3 uniquely classify any given system as either IC or not IC.

While it is known that the reverse direction of Theorem 3 does not hold, it is believed that the reverse direction of Theorem 2 does. In the absence of any proof or counterexample we state this as the following.

Conjecture 1: If there exists a diagonal matrix, $X$, such that $\sigma[G(0) X] \subset \overline{\mathcal{C}}_{+}-\{0\}$, then $G(s)$ is IC.

We close this section with the following result which relates systems which are IC to those which are IS.

Theorem 4: There exists a permutation matrix, $P$, such that $P G(s)$ is IC if and only if $G(s)$ is IS.

Proof: Given that $P G(s)$ is IC, we have from Theorem 2 that $P G(0)$ must be nonsingular. Since every permutation matrix is nonsingular, this implies that $G(0)$ is nonsingular, and therefore $G(s)$ is IS.

Given that $G(s)$ is IS, Theorem 1 implies that $G(0)$ is nonsingular. Using Gaussian elimination we can derive from $G(0)$ nonsingular matrices $P, L$, and $U$, where $P$ is a permutation matrix, $L$ a lower triangular matrix, and $U$ an upper triangular matrix such that $P G(0)=L U$. Elementary calculation then provides that

$$
\operatorname{det}[P G(0)]_{m_{r}^{l}}=\prod_{i=1}^{r} l_{i i} u_{i i}
$$

where $m_{r}^{l}$ denotes the leading principal submatrix of $P G(0)$ of dimension $r$. Since $L$ and $U$ are nonsingular it must hold that $l_{i i} \neq 0$ and $u_{i i} \neq 0$ for all $i \in\{1, \cdots, n\}$. It follows then that all leading principal submatrices of $P G(0)$ are nonsingular. By Corollary 1, this implies that the system $P G(s)$ is IC.

Remark:

The existence of a permutation such that $P G(s)$ is IC is equivalent to saying that there exists an input-output pairing such that $G(s)$ is $\mathrm{IC}$. This means that if $G(0)$ is nonsingular, we can always find at least one pairing for which there exists a controller which unconditionally stabilizes $G(s)$.

\section{Integral Controllability with Integrity}

Before developing conditions for ICI, we introduce some additional definitions.

Definition 9: The matrix $A$ is a $\mathcal{P}$-matrix if $\operatorname{det} A_{m}>0$ for all $m \in M$.

In the obvious way we define the set, $\mathcal{P}$, consisting of all $\mathcal{P}$-matrices,

$$
\mathcal{P} \triangleq\left\{A \mid \operatorname{det} A_{m}>0 \text { for all } m \in M\right\}
$$

so that we may write, $A \in \mathcal{P}$ to mean that $A$ is a $\mathcal{P}$-matrix.

We also introduce the notation, $A^{+}$, to denote the matrix derived from $A$ by postscaling to make its diagonal elements nonnegative.

Definition 10: Given the matrix $A$, we denote by $A^{+}$the matrix, $A^{+}=A S_{A}$, where the "sign matrix," $S_{A}$, is defined by $^{5}$

$$
S_{A} \triangleq \operatorname{diag}\left\{\operatorname{sign}\left(a_{11}\right), \operatorname{sign}\left(a_{22}\right), \cdots, \operatorname{sign}\left(a_{n n}\right)\right\}
$$

and for any real number, $x$,

$$
\operatorname{sign}(x) \triangleq\left\{\begin{array}{rl}
1 & \text { if } x \geq 0 \\
-1 & \text { if } x<0
\end{array} .\right.
$$

Finally we introduce the relative gain array (originally defined by Bristol [5]) a commonly used tool in the pairing selection problem.

Definition 11: Given a nonsingular matrix $A$, the relative gain array (RGA) is defined to be the matrix

$$
\Lambda(A) \triangleq A \otimes\left[A^{-1}\right]^{T}
$$

where $\otimes$ denotes element-by-element multiplication of matrices (often called the Hadamard or Schur product).

The diagonal elements of $\Lambda[G(0)]$ are commonly referred to as the relative gains of the system $G(s)$.

The following well-known properties of the RGA are easily proven (see, for example, [16]).

Lemma 2: For any nonsingular matrix $A$, the following hold:

1) $\lambda_{i j}(A)=\left(a_{i j} A^{i j} / \operatorname{det}(A)\right)$, where $A^{i j}$ is the $(i, j)$ th cofactor of $A^{6}$

2) $P_{1} \Lambda(A) P_{2}=\Lambda\left(P_{1} A P_{2}\right)$ for any permutation matrices $P_{1}$ and $P_{2}$.

3) $\Lambda(A)=\Lambda\left(X_{1} A X_{2}\right)$ for any nonsingualr diagonal matrices $X_{1}$ and $X_{2}$.

With these preliminaries we can prove the following result which leads directly to a necessary condition for ICI.

Lemma 3: For any matrix $A$ the following are equivalent:

1) $A^{+} \in \mathcal{P}$.

2) There exists a diagonal matrix, $X$, such that $A X \in \mathcal{P}$.

3) $\Lambda\left(A_{m}\right)$ exists and its diagonal elements are positive for all $m \in M$.

Proof: We show that 1) $\Rightarrow 2) \Rightarrow 3$ ) $\Rightarrow 1$ ).

By definition, $A^{+}=A S_{A}$, so that $A^{+} \in \mathcal{P}$ implies that there exists an $X$, namely $X=S_{A}$, such that $A X \in \mathcal{P}$. Thus 1) implies 2). Next we show that 2) implies 3). $A X \in \mathcal{P}$ implies that $A_{m}$ is nonsingular for all $m \in M$ so that existence of $\Lambda\left(A_{m}\right)$ is established. By Lemma 2, we have

$$
\begin{aligned}
\lambda_{i i}\left(A_{m}\right) & =\lambda_{i i}(A X)_{m} \quad \text { for all } m \in M \\
& =\frac{a_{i i} x_{i i}(A X)_{m}^{i i}}{\operatorname{det}(A X)_{m}} \quad \text { for all } m \in M .
\end{aligned}
$$

We see then that $\lambda_{i i}\left(A_{m}\right)$ consists of three factors, $a_{i i} x_{i i}$, $(A X)_{m}^{i i}$, and $1 / \operatorname{det}(A X)_{m}$, each of which is positive since $A X \in \mathcal{P}$. Thus 2 ) implies 3 ). Finally we show that 3 ) implies 1). We have

$$
\lambda_{i i}\left(A_{m}\right)>0 \text { for all } m \in M
$$

which implies

$$
\lambda_{i i}\left(A_{m}^{+}\right)>0 \text { for all } m \in M
$$

${ }^{5}$ Notice that by definition $S_{A}$ is nonsingular and $S_{A}^{-1}=S_{A}$ for any $A$. ${ }^{6}$ For $A$ of dimension 1 we take $A^{11}=1$ by convention. 
so that

$$
\frac{a_{i i}^{+}\left(A_{m}^{+}\right)^{i i}}{\operatorname{det}\left(A_{m}^{+}\right)}>0 \text { for all } m \in M
$$

Since $a_{i i}^{+}>0$ by construction, we must have

$$
\frac{\left(A_{m}^{+}\right)^{i i}}{\operatorname{det}\left(A_{m}^{+}\right)}>0 \text { for all } m \in M \text {. }
$$

Equation (16) allows us to prove that $A^{+} \in \mathcal{P}$ by induction. We first establish that $\operatorname{det}\left(A_{m_{1}}^{+}\right)>0$ for $\operatorname{dim}\left(m_{1}\right)=1$. Then assuming that $\operatorname{det}\left(A_{m_{r}}^{+}\right)>0$ for $\operatorname{dim}\left(m_{r}\right)=r$, we show that (16) implies that $\operatorname{det}\left(A_{m_{r+1}}^{+}\right)>0$ for $\operatorname{dim}\left(m_{r+1}\right)=r+1$. This completes the induction and establishes that $\operatorname{det}\left(A_{m_{r}}^{+}\right)>$ 0 for all $\operatorname{dim}\left(m_{r}\right)=r \in\{1, \cdots, n\}$, or equivalently that $A^{+} \in \mathcal{P}$.

Consider first the case that $r=1$. The principal submatrices of $A^{+}$of dimension 1 are simply its diagonal elements, $a_{i i}^{+}$. Since these are positive by construction of $A^{+}$, we have $\operatorname{det}\left(A_{m_{1}}^{+}\right)>0$ for all $m \in M$ such that $\operatorname{dim}\left(m_{1}\right)=1$.

We now assume that for some fixed $r>1, \operatorname{det}\left(A_{m_{r}}^{+}\right)>0$ holds for all $m_{r} \in M$. This assumption implies that the diagonal cofactors of $A_{m_{r+1}}^{+}$, denoted $\left(A_{m_{r+1}}^{+}\right)^{i i}$, are positive since these cofactors are simply the determinants of principal submatrices of $A_{m_{r+1}}^{+}$of dimension $r$, i.e., $\left(A_{m_{r+1}}^{+}\right)^{i i}=$ $\operatorname{det}\left(A_{m_{r}}^{+}\right)>0$. With $\left(A_{m_{r+1}}^{+}\right)^{i i}>0$ for all $i$, (16) implies that $\operatorname{det}\left(A_{m_{r+1}}^{+}\right)>0$ for all $m_{r+1} \in M$. The induction is then established, and it must hold that $\operatorname{det}\left(A_{m_{r}}^{+}\right)>0$ for all $m_{r} \in M$ such that $r \in\{1, \cdots, n\}$. This is equivalent to the condition $A_{m}^{+} \in \mathcal{P}$ for all $m \in M$. Thus we have established that 3 ) implies 1 ), and the proof is complete. ICI.

We are now in a position to state a necessary condition for

Theorem 5: $G(s)$ is ICI only if $G(0)$ satisfies any (and hence all) of the conditions of Lemma 3.

Proof: Using Fact 4, $G(s)$ ICI implies that there exists a controller, $K(s)=(1 / s) C(s)$, such that $K_{m}(s)$ stabilizes $G_{m}(s)$ for all $m \in M$. This implies by Theorem 1 that $G_{m}(0)$ must be nonsingular for all $m \in M$. Furthermore by Fact 1 we must have

$$
Q_{\text {sub }}(s)=K_{m}(s)\left[I+G_{m}(s) K_{m}(s)\right]^{-1}
$$

stable for all $m \in M$. With $G(s)$ stable this implies that

$$
I-G_{m}(s) Q_{\mathrm{sub}}(s)=\left[I+G_{m}(s) K_{m}(s)\right]^{-1}
$$

must be stable for all $m \in M$. This implies in turn that the polynomial equation

$$
\operatorname{det}\left[I+G_{m}(s) K_{m}(s)\right]=0
$$

has no roots in $\overline{\mathcal{C}}_{+}$. Substituting $G_{m}(s) K_{m}(s)=\left(1 / s d_{m}(s)\right)$ $N_{m}(s)$ where

$$
d_{m}(s)=a_{i} s^{i}+\cdots+a_{1} s+1
$$

is the (stable) pole polynomial of $G_{m}(s) C_{m}(s)$, and $N_{m}(s)$ is a polynomial matrix, we have

$$
\frac{1}{s^{r} d_{m}^{r}(s)} \operatorname{det}\left[s d_{m}(s) I+N_{m}(s)\right]=0
$$

where $r=\operatorname{dim}(m)$. Since $d_{m}(s)$ is nonzero on $\overline{\mathcal{C}}_{+},(21)$ will have no roots in $\overline{\mathcal{C}}_{+}$only if

$$
\operatorname{det}\left[s d_{m}(s) I+N_{m}(s)\right]=0
$$

has no roots in $\overline{\mathcal{C}}_{+}-\{0\}$. Expanding the determinant in (22) we have

$$
s^{r} d_{m}^{r}(s)+\cdots+\operatorname{det}\left[N_{m}(0)\right]=0 .
$$

If this polynomial is to have no roots in $\overline{\mathcal{C}}_{+}-\{0\}$ then by Routh-Hurwitz, all coefficients in (23) must be nonnegative. This implies that

$$
\operatorname{det}\left[N_{m}(0)\right]=\operatorname{det}\left[G_{m}(0) C_{m}(0)\right] \geq 0 .
$$

We have $\operatorname{det} C_{m}(0) \neq 0$ by assumption and we have previously shown that $\operatorname{det} G_{m}(0) \neq 0$ so that we require

$$
\operatorname{det}\left[G_{m}(0) C_{m}(0)\right]>0
$$

for all $m \in M$. This implies that there exists a diagonal matrix, $X=C(0)$, such that $G(0) X \in \mathcal{P}$ and the result is proven.

Remarks:

1) Theorems 3.3 and 3.4 of [7] can be reworked to form a necessary condition for ICI equivalent to condition 3 ) of Lemma 3.

2) The reverse direction of Theorem 5 holds for $3 \times 3$ and smaller systems (we state and prove this in Section VI below). It is conjectured that the reverse direction does not hold in general although no proof of counterexample has been demonstrated.

3) A somewhat surprising consequence of Theorem 5 is the following corollary.

Corollary 2: $G(s)$ is integral controllable with integrity only if $G(s)$ is integral controllable.

Proof: $G(s)$ ICI implies that $G^{+}(0) \in \mathcal{P}$ which implies that all of the principal submatrices of $G(0)$ are nonsingular. By Corollary 1 this implies that $G(s)$ is IC.

This result says that if there exists a controller which together with $G(s)$ forms a closed-loop system with integrity that there must also exist a controller which together with $G(s)$ forms an unconditionally stable closed loop. This is somewhat unexpected since the sets $\mathcal{E}_{1 / 0}$ and $\mathcal{E}_{I}$ are unrelated.

4) An obvious corollary to Theorem 5 is the condition implicit in Theorem 6 of [26] that the relative gains of $G(s)$ must be positive for integral controllability with integrity.

Corollary 3: $G(s)$ is integral controllable with integrity only if $\lambda_{i i}[G(0)]>0, i=1, \cdots, n$.

While it is true that positive relative gains are necessary for controllability with integrity, this condition is not sufficient. In fact, as the following example shows, positive relative gains are not even sufficient for the weaker condition that $G(s)$ be IC.

Example 1: Let $G(s)$ be given such that

$$
G(0)=\left[\begin{array}{cccc}
1 & 1 & 1 & 1 \\
1 & 1 & \frac{1}{2} & 2 \\
\frac{1}{4} & \frac{1}{2} & \frac{1}{4} & 4 \\
4 & 2 & \frac{1}{4} & 4
\end{array}\right]
$$


which has positive relative gains, $\lambda_{i i} \in$ $\{.800,5.00, .178, .178\}$. By construction, every $2 \times 2$ principal submatrix of $G(0)$ is singular, so that $G^{+}(0) \notin \mathcal{P}$ and $G(s)$ is not ICI. Furthermore it can be shown using the Routh-Hurwitz criteria that there exists no diagonal $X$ such that $\sigma[G(0) X] \subset \overline{\mathcal{C}}_{+}-\{0\}$. Thus by Theorem $2, G(s)$ is not IC.

5) Chang and Davison [6] state as Theorem 1 of their paper that $G^{+}(0) \in \mathcal{P}$ is necessary and sufficient for the existence of a controller, $K(s)=(1 / s) X$, with $X$ a nonsingular constant diagonal matrix such that the following hold:

a) $K(s)$ unconditionally stabilizes $G(s)$.

b) Every SISO principal subsystem of $K(s)$ stabilizes the corresponding principal subsystem of $G(s)$.

That $G^{+}(0) \in \mathcal{P}$ is not necessary is easily shown with the following example.

Example 2: Let

$$
G(s)=\frac{1}{s+1}\left[\begin{array}{lll}
1 & 1 & 1 \\
1 & 1 & 2 \\
1 & 0 & 1
\end{array}\right]
$$

$G(0)$ is clearly not a $\mathcal{P}$-matrix since $G_{m}(0)$ is singular for $m=\{1,2\}$. However, $\sigma[G(0)]=\{.1154 \pm$ $.5897 i, 2.769\} \subset \mathcal{C}_{+}$, so that by Fact 3 there exists an $\gamma>0$ such that $K(s)=(\gamma / s)$ unconditionally stabilizes $G(s)$. That $K_{m}(s)$ also stabilizes $G_{m}(s)$ when $\operatorname{dim}(m)=1$ is also clear. Thus the required condition, $G^{+}(0) \in \mathcal{P}$, cannot be necessary.

We note that the condition $G^{+}(0) \in \mathcal{P}$ is sufficient for properties a) and b) to hold. In fact it can be shown using Lemma 1 and Fact 3 that the weaker condition there exists a permutation matrix, $P$, such that all leading principal submatrices of $P G^{+}(0) P^{T}$ have positive determinants, is sufficient for the stated properties to hold.

A sufficient condition for $G(s)$ to be integral controllable with integrity is given by the following theorem.

Theorem 6: If there exists a diagonal matrix $X$ such that $\sigma[G(0) X]_{m} \subset \mathcal{C}_{+}$for all $m \in M$, then $G(s)$ is ICI.

Proof: By Theorem 3, under the given conditions for every $m \in M$ there exists a $\gamma(m)>0$ sufficiently small such that the controller $K_{m}(s)=(\gamma(m) / s) X_{m}$ unconditionally stabilizes $G_{m}(s)$ for all $m \in M$. By taking $\gamma^{*}=$ $\min _{m \in M} \gamma(m)$, we define the controller $K(s)=\left(\gamma^{*} / s\right) X$ which, by construction, demonstrates that $G(s)$ is ICI.

Remarks:

1) The condition of Theorem 6 implies not only that $G(s)$ is ICI, but also that there exists a pure integral controller, $K(s)=\left(\gamma^{*} / s\right) X$, with $X$ a constant matrix, which together with $G(s)$ forms a closed-loop system with integrity.

2) In light of Remark 1) it is conjectured that the reverse direction of Theorem 6 does not hold although no specific counterexample has been demonstrated.

\section{DECENTRALIZED INTEGRAL CONTROLLABILITY}

Preliminary to the study of systems which are DIC, we introduce the notion of $D$-stability, which was originally defined by Arrow and McManus [2]. The definition currently in use in the literature is slightly less restrictive than Arrow and McManus' and is adopted here.

Definition 12: The real matrix $A$ is $D$-stable if and only if $\sigma(A D) \subset \mathcal{C}_{+}$for all diagonal matrices $D>0$.

For convenience we define the set of all $D$-stable matrices

$$
D L \triangleq\left\{A \mid \sigma(A D) \subset \mathcal{C}_{+} \forall \text { diagonal } D>0\right\}
$$

and the related set

$$
\overline{D L} \triangleq\left\{A \mid \sigma(A D) \subset \overline{\mathcal{C}}_{+} \forall \text { diagonal } D>0\right\} .
$$

With a slight abuse of notation, we will write $A \in \overline{D L}-\{0\}$ to indicate that $A$ is nonsingular in addition to being in the set $\overline{D L}$, i.e., $A \in \overline{D L}-\{0\}$ if and only if $\sigma(A D) \subset \overline{\mathcal{C}}_{+}-\{0\} \forall$ diagonal $D>0$. With these ideas understood, we can state the following necessary condition for the system $G(s)$ to be DIC.

Theorem 7: $G(s)$ is DIC only if $G_{m}^{+}(0) \in \overline{D L}-\{0\}$ for all $m \in M$.

Proof: By Fact 5, $G(s)$ is DIC if and only if there exists a $K(s)=(1 / s) C(s)$ with $C(s)$ diagonal and $C(0)$ nonsingular such that $\left(1 /\|D\|_{2}\right)[D K(s)]_{m}$ unconditionally stabilizes $G_{m}(s)$ for all $m \in M$ and for all diagonal $D>0$. Using Fact 2 this implies that

$$
\sigma\left\{\left[\frac{1}{\|D\|_{2}} G(0) D C(0)\right]_{m}\right\} \subset \overline{\mathcal{C}}_{+}-\{0\}
$$

for all $m \in M$, and $D>0$. This implies that

$$
\sigma\left\{[G(0) D C(0)]_{m}\right\} \subset \overline{\mathcal{C}}_{+}-\{0\}
$$

for all $m \in M$, and $D>0$. Writing $G(0)$ and $C(0)$ as

$$
\begin{aligned}
& G(0)=G^{+}(0) S_{G(0)}^{-1}=G^{+}(0) S_{G(0)} \\
& C(0)=C^{+}(0) S_{C(0)}^{-1}=S_{C(0)} C^{+}(0)
\end{aligned}
$$

and substituting into $(31)$ provides

$$
\sigma\left\{\left[G^{+}(0) S_{G(0)} S_{C(0)} C^{+}(0) D\right]_{m}\right\} \subset \overline{\mathcal{C}}_{+}-\{0\}
$$

for all $m \in M$, and $D>0$. For (31) to hold with $D=I$ and $\operatorname{dim}(m)=1$, it must hold that $g_{i i}(0) c_{i i}(0)>0$ for all $i \in\{1, \cdots, n\}$. It follows then that $S_{G(0)} S_{C(0)}=I$. Thus if (34) is to hold, we must have $\sigma\left\{\left[G^{+}(0) \hat{D}\right]_{m}\right\} \subset \overline{\mathcal{C}}_{+}-\{0\}$ for all $m \in M$, where $\hat{D}=C^{+}(0) D$ is an arbitrary positive definite diagonal matrix. This implies by definition that $G_{m}^{+}(0) \in \overline{D L}-\{0\}$ for all $m \in M$.

\section{Remarks:}

1) All known necessary conditions for $G(s)$ to be DIC follow from Theorem 7 as special cases. Specific examples include the following.

Corollary 4 [27]: $G(s)$ is DIC only if both of the following hold:

a) $\sigma\left[G^{+}(0) D\right] \subset \overline{\mathcal{C}}_{+}$for all diagonal $D \geq 0$.

b) The relative gains of $G(s)$ are positive. 
Proof: Condition a) follows trivially from Theorem 7. Condition b) is established as follows. By Theorem $7, G(s)$ is DIC implies that $G_{m}^{+}(0) \in \overline{D L}-\{0\}$ for all $m \in M$. It follows immediately that $G^{+}(0) \in \mathcal{P}$. By Lemma 3 this implies that the relative gains of $G^{+}(s)$ must be positive which, in turn, implies that the relative gains of $G(s)$ must be positive.

2) The condition of Theorem 7 (along with a) of Corollary 4) is not finitely verifiable so that it is not useful as a practical test for $G(s)$ to be DIC. The best computable necessary condition obtainable from Theorem 7 is apparently that the spectrum of $G^{+}(0) D$ and all of its principal submatrices must lie in $\overline{\mathcal{C}}_{+}-\{0\}$ for a specific $D>0$ (or a finite number of such $D$ s). For example, with $D=I$ Theorem 7 becomes the following.

Corollary 5: $G(s)$ is DIC only if $\sigma\left\{\left[G^{+}(0)\right]_{m}\right\} \subset$ $\overline{\mathcal{C}}_{+}-\{0\}$ for all $m \in M$. Note that this implies, but is not implied, by $G^{+}(0) \in \mathcal{P}$, a necessary condition for $G(s)$ to be integral controllable with integrity.

3) It is conjectured that the reverse direction of Theorem 7 does not hold although no proof or counterexample is known.

The concept of $D$-stability can also be used to develop a very general sufficient condition for DIC.

Theorem 8: If $G_{m}^{+}(0) \in D L$ for all $m \in M$, then $G(s)$ is DIC.

Proof: It follows from the given condition that $\sigma\{[G(0)$ $\left.\left.S_{G(0)} D\right]_{m}\right\} \subset \mathcal{C}_{+}$for all $m \in M$ and all diagonal $D>0$. This implies that there exists a nonsingular matrix $X$, namely $X=S_{G(0)}$, such that $\sigma[G(0) D X]_{m} \subset \mathcal{C}_{+}$for all $m \in M$ and all $D>0$. It follows from Theorem 3 (Remark 1)) that for all $m \in M$ and for all diagonal $D>0$ there exists a $\gamma>0$ such that $K_{m}(s)=(\gamma / s) X_{m}$ unconditionally stabilizes $[G(s) D]_{m}$. By Fact 4 and Definition 3 this implies that for all diagonal $D>0$ and for all $E \in \mathcal{E}_{1 / 0}, \hat{K}(s)=(\gamma / s) E X$ stabilizes $G(s) D$ This, in turn, implies that for all diagonal $D>0$ and for all $E \in \mathcal{E}_{1 / 0}, \hat{\hat{K}}(s)=(\gamma / s) D E X$ stabilizes $G(s)$. Since the set of all $\hat{E}=D E$ where $D$ is positive definite and $E \in \mathcal{E}_{1 / 0}$ contains $\mathcal{E}_{D}$, this implies that $G(s)$ is DIC.

Remarks:

1) The condition of Theorem 8 implies not only that $G(s)$ is DIC, but also that there exists a pure integral controller of the form, $K(s)=(\gamma / s) X$, with $X$ a constant matrix, which demonstrates that $G(s)$ is DIC.

2) In light of Remark 1), it is not surprising that the condition of Theorem 8 is known to not be necessary for DIC. We demonstrate this with the following example. Example 3: For the plant

$$
G(s)=\left[\begin{array}{ccc}
1 & 0 & 2 \\
\frac{1}{s+1} & 1 & \frac{-4 s}{s+1} \\
0 & 4 & 1
\end{array}\right]
$$

it can be shown that the controller, $K(s)=(1 / s) I$, results in a decentralized unconditionally stable closedloop system. ${ }^{7}$ Thus, by construction, $G(s)$ is DIC. The

${ }^{7}$ This involves a straightforward but tedious application of the Routh-Hurwitz stability criteria. eigenvalues of $G(0)$ are $\sigma[G(0)]=\{ \pm \sqrt{3} i, 3\}$ so that $G(0)$ is not $D$-stable.

Note that although $G(0)$ has eigenvalues on the imaginary axis, $\sigma[G(0) D]_{m} \subset \overline{\mathcal{C}}_{+}-\{0\}$ for all $D>0$ and for all $m \in M$ so that the necessary conditions of Theorem 7 are fulfilled. As in the tests for IC, the gap between the necessary conditions of Theorem 7 and the sufficient conditions of Theorem 8 occurs along the imaginary axis-when $G^{+}(0)$ and its principal submatrices are in $\bar{D} \bar{L}\{0\}$ but not in $D L$.

3) In Theorems 1 and 2 of [30], Nwokah et al. claim that the condition of Theorem $8, G_{m}(0) \in D L$ for all $m \in M$, is necessary both for $K(s)=(1 / s) I$ with $G(s)$ to provide decentralized unconditional stability, and for $G(s)$ to be DIC.

Example 3 above demonstrates that this condition cannot be necessary for either decentralized unconditional stability or DIC since $K(s)=(1 / s) I$ and $G(s)$ constitute a decentralized unconditionally stable closed loop while $G(0)$ is not $D$-stable.

A complete characterization of matrices for which $A_{m} \in$ $D L$ for all $m \in M$ is provided by Hartfiel [19].

Lemma 4: Given the $n \times n$ matrix $A$, with $\sigma\left(A_{m}\right) \subset \mathcal{C}_{+}$ for all $m \in M$, the following are equivalent:

1) $A_{m} \in D L$ for all $m \in M$.

2) $A$ is interior to the set $D L$.

3) $p(D, \dot{A})>0$ for all diagonal $D>0$ where

$$
\begin{aligned}
p(D, A) & =\operatorname{det}\left[I+(D A)^{2}\right] \\
& =\sum_{\substack{\alpha, \beta \in M \\
\operatorname{dim}(\alpha)=\operatorname{dim}(\beta)=0,1, \cdots, \mathrm{n}}} \operatorname{det}\left[A_{\alpha, \beta} A_{\beta, \alpha} D_{\alpha, \alpha} D_{\beta, \beta}\right]
\end{aligned}
$$

where $A_{\alpha, \beta}$ denotes the (not necessarily principal) submatrix of $A$ with rows indexed by $\alpha$ and columns indexed by $\beta .^{8}$

In the vocabulary of [1], the condition that $A$ lie in the topological interior of $D L$ is equivalent to the statement that $A$ is strongly $D$-stable. Using this result we can restate Theorem 8 as Corollary 6.

Corollary 6: If for all $m \in M, \sigma\left[G_{m}^{+}(0)\right] \subset \mathcal{C}_{+}$and $G_{m}^{+}(0)$ satisfies any (and hence all) of the conditions of Lemma 4 , then $G(s)$ is DIC.

While Lemma 4 provides necessary and sufficient conditions on $G^{+}(0)$ such that the condition of Theorem 8 is satisfied, the condition of the resulting Corollary 6 is no more easily verified than that of the theorem. On the other hand any condition which implies that $G_{m}^{+}(0) \in D L$ for all $m \in M$ trivially generates another (possibly conservative) Corollary to Theorem 8. For an overview of available results of this kind, the interested reader is referred to the excellent survey by Johnson [20] and references therein.

Among the known conditions which imply that all principal submatrices of a given matrix are $D$-stable, we will focus on the following.

${ }^{8}$ By convention we take the determinant of an empty matrix (zero rows or columns) to be one. 
Lemma 5: The following conditions are equivalent and imply that $A_{m} \in D L$ for all $m \in M$.

1) There exists a diagonal $F>0$ such that $A F+F A^{T}>0$.

2) $(I+A)^{-1}$ exists and there exists a diagonal $F>0$ such that $\left\|F(I-A)(I+A)^{-1} F^{-1}\right\|_{2}<1$.

Proof: That 1) implies $A \in D L$ is due to Arrow and McManus [2]. The extension to $A_{m} \in D L$ for all $m \in M$ can be found in [20]. See also [4] for a discussion. It remains to show that 1 ) is equivalent to 2 ). We first note that 1 ) implies that $\sigma(A) \subset \mathcal{C}_{+}$by Lyaponov's theorem so that $(I+A)^{-1}$ must exist. Given existence of $(I+A)^{-1}$, it follows that:

$$
\begin{aligned}
\| & F(I-A)(I+A)^{-1} F^{-1} \|_{2}<1 \\
\Leftrightarrow & \lambda_{\max }\left[\left(I+F A F^{-1}\right)^{-T}\left(I-F A F^{-1}\right)^{T}\right. \\
& \left.\cdot\left(I-F A F^{-1}\right)\left(I+F A F^{-1}\right)^{-1}\right]<1 \\
\Leftrightarrow & \left(I+F A F^{-1}\right)^{-T}\left(I-F A F^{-1}\right)^{T} \\
& \cdot\left(I-F A F^{-1}\right)\left(I+F A F^{-1}\right)^{-1}<I \\
\Leftrightarrow & \left(I-F A F^{-1}\right)^{T}\left(I-F A F^{-1}\right) \\
< & \left(I+F A F^{-1}\right)^{T}\left(I+F A F^{-1}\right) \\
\Leftrightarrow & -\left(F A F^{-1}\right)^{T}-F A F^{-1}<\left(F A F^{-1}\right)^{T}+F A F^{-1} \\
\Leftrightarrow & F A F^{-1}+\left(F A F^{-1}\right)^{T}>0 \\
\Leftrightarrow & A F^{-2}+F^{-2} A^{T}>0
\end{aligned}
$$

and the proof is complete.

Lemma 5 together with Theorem 8 provides immediately the following corollary.

Corollary 7: If $G^{+}(0)$ satisfies either (hence both) of the conditions of Lemma 5 , then $G(s)$ is DIC.

Remarks:

1) Khalil [21] provides an algorithm which given any $G^{+}(0)$ is guaranteed to find an $F$ satisfying condition 1) of Lemma 5 if one exists. A practical drawback is that the algorithm need not terminate in finite time. A more attractive numerical approach is to solve

$$
\min _{F>0 \text {, diagonal }}\left\|F\left[I-G^{+}(0)\right]\left[I+G^{+}(0)\right]^{-1} F^{-1}\right\|_{2}
$$

to satisfy condition 2) of Lemma 5. Much research has focused on the solution of this optimization problem in connection with the evaluation of an upper bound on the structured singular value [11], and several algorithms are available for its solution (see, e.g., $[12,32]$ ). These algorithms make use of the fact that (45) can be reformulated as a convex optimization problem for which descent directions are provided by the solution of an eigenvalue problem.

2) A significant advantage of Corollary 7 is that since it involves a single test on $G^{+}(0)$, no further examination of the principal submatrices of $G^{+}(0)$ is required. This is particularly important for large systems since the number of principal submatrices grows exponentially with $n$.

3) A disadvantage of Corollary 7 compared to Corollary 6 is that it is more conservative. Hartfiel [19] provides a $3 \times 3$ example with $A_{m} \in D L$ for all $m \in M$ for which no $F$ exists to satisfy 1 ) of Lemma 5 .
4) The most obvious consequence of Corollary 7 is that any system which has an (adjusted) steady-state gain matrix, $G^{+}(0)$, with positive definite symmetric component is DIC. ${ }^{9}$ Although significantly more restrictive than Theorem 8 , or even Corollary 7 , this previously unknown, and obviously simple, condition has proven useful in several practically motivated examples. ${ }^{10}$

A number of simple sufficient conditions for DIC follow from Corollary 7 and are stated in terms of the "error system," $L(s)$, defined (whenever it exists) by

$$
L(s)=[G(s)-\bar{G}(s)] \bar{G}^{-1}(s)
$$

where $\bar{G}(s) \triangleq \operatorname{diag}\left\{g_{i i}(s)\right\}$. The norm of $L(s)$ is a measure of the relative error incurred by approximating $G(s)$ by its diagonal elements.

Using Corollary 7 we can easily prove the following.

Corollary 8: If $L(0)$ exists and

$$
\min _{F>0, \text { diagonal }}\left\|F L(0) F^{-1}\right\|_{p}<1
$$

for any $p \in\{1,2, \infty\}$, then $G(s)$ is DIC.

Proof: By the Perron-Frobenius Theorem, which states that for $p \in[1, \infty]$

$$
\rho(|L(0)|) \geq \min _{F>0, \text { diagonal }}\left\|F L(0) F^{-1}\right\|_{p}
$$

and that equality holds for $p=1$ and $p=\infty$, we conclude that

$$
\rho(|L(0)|)=\min _{F>0, \text { diagonal }}\left\|F L(0) F^{-1}\right\|_{1 \text { or } \infty}<1
$$

implies that

$$
\min _{F>0 \text {, diagonal }}\left\|F L(0) F^{-1}\right\|_{2}<1 .
$$

It remains then only to show that condition (50) implies that $G(s)$ is DIC. By the triangle inequality we have

$$
\min _{F>0 \text {, diagonal }}\left\|-F L(0) F^{-1}-F^{-1} L^{T}(0) F\right\|_{2}<2 .
$$

This implies that

$$
\mu_{2}\left[-F L(0) F^{-1}-F^{-1} L^{T}(0) F\right]<2
$$

where $\mu_{2}(\cdot)$ denotes the measure of its matrix argument defined by the induced 2-norm. (See $[9,10]$ for a definition of $\mu_{p}(\cdot)$ and a discussion of its properties). Using the definition of $L(0)$, this implies

$$
\mu_{2}\left[-F G(0) \bar{G}^{-1}(0) F^{-1}-F^{-1} \bar{G}^{-1}(0) G^{T}(0) F\right]<0
$$

which in turn implies that

$$
G(0) \bar{G}^{-1}(0) F^{-2}+F^{-2} \bar{G}^{-1}(0) G^{T}(0)>0 .
$$

Since $G(0) \bar{G}^{-1}(0)=\left[G(0) \bar{G}^{-1}(0)\right]^{+}$this implies by Corollary 7 that the system, $G(s) \bar{G}^{-1}(0)$ is DIC. This in turn implies that $G(s)$ is DIC, and the proof is complete.

${ }^{9}$ Recall that the symmetric component of the real matrix $A$, denoted $S(A)$, is given by $S(A)=(1 / 2)\left(A+A^{T}\right)$.

${ }^{10} \mathrm{See}$, for example, those in [23]-[25], [29], [33]. Example 5 of [33] is trivially shown to be DIC using this condition, while in the referenced paper an intensive numerical search is only able to provide that DIC is "extremely likely." 


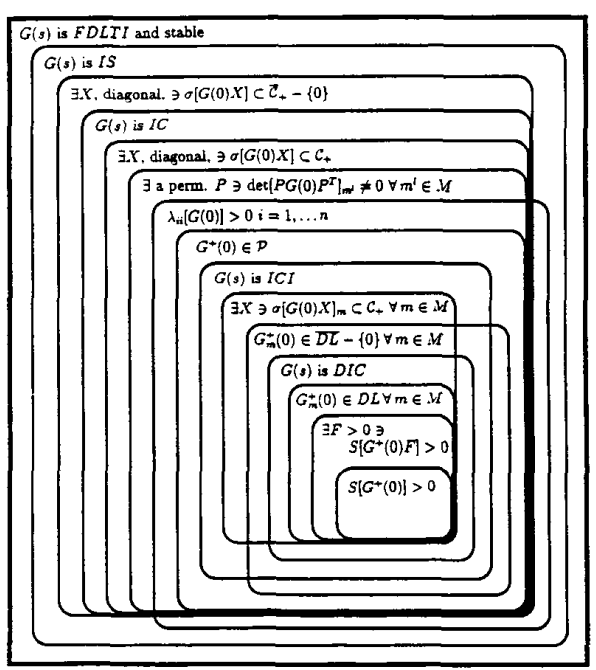

Fig. 2. Classification of general systems.

It follows from Corollary 8 that if $G(0)$ is (generalized) diagonally dominant, i.e,. $\rho(|L(0)|)<1$, then $G(s)$ is DIC. ${ }^{11}$

This is not surprising since diagonally dominant systems are "weakly coupled" in a precise mathematical sense. As we argued heuristically in the introduction, we expect weakly coupled systems to admit decentralized controllers with attractvie closed-loop properties.

What is more interesting is that the system $G(s)$ need not be weakly coupled (diagonally dominant) to be DIC. Indeed, as the next example shows, Corollary 8 can be arbitrarily conservative relative to Corollary 7 (which is itself more conservative than Theorem 8 ).

Example 4: Consider any $G(s)$ such that

$$
G(0)=\left[\begin{array}{cc}
1 & a \\
-a & 1
\end{array}\right]
$$

where $a \in \mathcal{R}$ is given. Since for any $a$

$$
G^{+}(0)+\left[G^{+}(0)\right]^{T}=I>0
$$

$G(s)$ is DIC. For $p \in\{1,2, \infty\}$, however,

$$
\min _{F>0, \text { diagonal }}\left\|F L(0) F^{-1}\right\|_{p}=|a|
$$

which can be made arbitrarily large by the choice of $a$.

We conclude this section on the classification of open-loop systems by summarizing all of the proven results in the Venn diagram of Fig. 2. Some nontrivial regions of the diagram are not currently known to be nonempty. For example, there is no known $G(s)$ which satisfies $G_{m}^{+}(0) \in \overline{D L}-\{0\}$ for all $m \in M$ yet is not DIC. Nonetheless, the indicated set inclusions are known to hold and the diagram facilitates the visualization of the known results.

\section{SPECialized Results for $2 \times 2$ AND $3 \times 3$ SystemS}

A number of simplifications of the results in Section $V$ are available for low dimensional systems. We collect these here in the following theorems.

\footnotetext{
${ }^{11}$ This result also follows from the development in [27].
}

Theorem 9: For $G(s)$ of dimension 2, the following are equivalent.

1) $\lambda_{11}[G(0)]>0$.

2) $G^{+}(0) \in \mathcal{P}$.

3) $G(s)$ is DIC.

4) $G(s)$ is ICI.

Proof: We prove that 1) $\Rightarrow 2$ ) $\Rightarrow 3$ ) $\Rightarrow 4$ ) $\Rightarrow 1$ ).

That 1$) \Rightarrow 2$ ) is evident from Lemma 2 which provides

$$
\lambda_{11}[G(0)]=\lambda_{11}\left[G^{+}(0)\right]=\frac{g_{11}^{+} g_{22}^{+}}{\operatorname{det} G^{+}(0)} .
$$

In the $2 \times 2$ case, $G^{+}(0) \in \mathcal{P}$ implies that $G_{m}^{+}(0) \in D L$ for all $m \in M$ [20], so that by Theorem 8,2) implies 3). That 3) implies 4) is trivial from the definitions of ICI and DIC. That 4) implies 1) follows from Theorem 5 .

In [34], Yu and Fan claim to provide a necessary and sufficient condition for DIC in the $3 \times 3$ case. Unfortunately the proof contains a flaw. We restate their result here in corrected form as Parts 1) and 2) of Theorem $10 .{ }^{12}$ Part 3) of Theorem 10 is a new result.

Theorem 10: For $G(s)$ of dimension 3,

1) If $G(s)$ is DIC, then $G^{+}(0) \in \mathcal{P}$ and $\sum_{i=1}^{3} \lambda_{i i}^{1 / 2}[G(0)] \geq 1$.

2) If $G^{+}(0) \in \mathcal{P}$ and $\sum_{i=1}^{3} \lambda_{i i}^{1 / 2}[G(0)]>1$, then $G(s)$ is DIC.

3) $G(s)$ is ICI if and only if $G^{+}(0) \in \mathcal{P}$.

Before stating the proof of Theorem 10, we note that according to Theorem 2 of [34], condition 2) is necessary as well as sufficient for $G(s)$ to be DIC. That this condition cannot be necessary is established by Example 3 for which $G^{+}(0) \in \mathcal{P}$ and $\sum_{i=1}^{3} \lambda_{i i}^{1 / 2}[G(0)]=1$ although $G(s)$ is DIC. A careful reworking of the arguments in the proof presented in [34], however, provides the following result.

Lemma 6: Let $A$ be a $3 \times 3$ matrix with $A^{+} \in \mathcal{P}$, then the following hold:

1) 1$) A_{m}^{+} \in \overline{D L}-\{0\}$ for all $m \in M$ implies $\sum_{i=1}^{3} \lambda_{i i}^{1 / 2}(A) \geq 1$.

2) $\sum_{i=1}^{3} \lambda_{i i}^{1 / 2}(A)>1$ implies $A_{m}^{+} \in D L$ for all $m \in M$. Proof: We first note that for $\operatorname{dim}(m) \leq 2, A^{+} \in \mathcal{P}$ imlies that $A_{m}^{+} \in D L$ (and hence $A_{m}^{+} \in \overline{D L}-\{0\}$ as well) [20]. It remains to show that in the $3 \times 3$ case

$$
\begin{aligned}
& \sigma\left(A^{+} D\right) \subset \overline{\mathcal{C}}_{+}-\{0\} \\
& \quad \text { for all diagonal } D>0 \Rightarrow \sum_{i=1}^{3} \lambda_{i i}^{1 / 2}(A) \geq 1
\end{aligned}
$$

and

$\sum_{i=1}^{3} \lambda_{i i}^{1 / 2}(A)>1 \Rightarrow \sigma\left(A^{+} D\right) \subset \overline{\mathcal{C}}_{+}-\{0\}$

for all diagonal $D>0$.

The desired result is obtained by following Yu and Fan, and applying the Routh-Hurwitz criteria to determine the location of the spectrum of $A^{+} D$ for arbitrary $D$.

\footnotetext{
${ }^{12}$ Unfortunately the corrected version no longer provides a necessary and sufficient condition.
} 


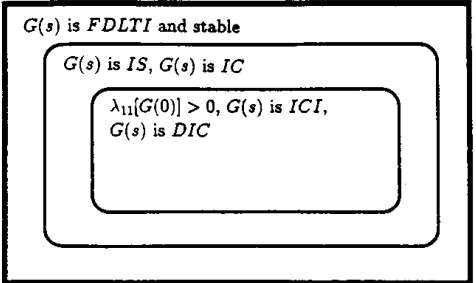

Fig. 3. Classification of $2 \times 2$ systems.

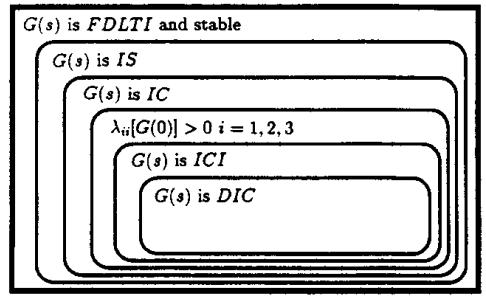

Fig. 4. Classification of $3 \times 3$ systems.

We will continue with the proof of Theorem 10.

Proof (of Theorem 10]: Using Lemma 6, parts 1) and 2) of Theorem 10 follow trivially from Theorems 7 and 8 , respectively. Part 3 ) remains to be shown. Given that $G(s)$ is ICI, it holds that $G^{+}(0) \in \mathcal{P}$ by Theorem 5 . This implies (by Lemma 1) that there exists a diagonal $F>0$ such that $\sigma\left[G^{+}(0) F\right] \subset \mathcal{C}_{+}$. Furthermore, $G^{+}(0) \in \mathcal{P}$ and $F>0$ diagonal imply that $G^{+}(0) F \in \mathcal{P}$ This implies that $\sigma\left[G^{+}(0) F\right]_{m} \subset \mathcal{C}_{+}$for all $m \in M$. (Any $1 \times 1$ principal submatrix of $G^{+}(0) F$ is positive by the definition of $G^{+}(0)$ and the fact that $F>0$. Any $2 \times 2$ principal submatrix must have its spectrum in the open right-half plane since both its trace and its determinant are positive). Thus there exists a nonsingular matrix, $X=S_{G(0)} F$, such that $\sigma[G(0) X]_{m} \subset \mathcal{C}_{+}$ for all $m \in M$. By Theorem 6 this implies that $G(s)$ is ICI.

As in the general $n \times n$ case these results are summarized in a Venn diagrams shown in Figs. 3 and 4.

\section{CONCLUSION}

Practically motivated classifications of a closed-loop and open-loop systems have been defined. The question of the existence of a decentralized controller which provides a closed loop with desired characteristics is studied in detail. The results here generalize and relate all known conditions, necessary or sufficient, for IS, IC, ICI, and DIC. The important connection between $D$-stability and DIC is demonstrated, and several examples are provided to illustrate this connection.

In addition to the independent theoretical interest of these results, we have laid the foundation for the development of a practical solution to the decentralized control variable pairing problem. Based on steady state gain information we can (essentially) determine if there exists a controller with integral action which will provide a closed-loop system with desired flexibility characteristics, any one or more of unconditional stability, integrity, or decentralized unconditional stability.
Among the screening tools for variable pairing obtainable from the results of this paper, the most practically relevant are:

1) The necessary condition for DIC of Corollary 5.

2) The necessary condition for DIC of Theorem 5 based on Condition 3) of Lemma 3.

3) The sufficient condition for DIC of Corollary 7 based on Condition 2) of Lemma 5.

Condition 1) is apparently the tightest known computable condition necessary for DIC. Unfortunately it requires the evaluation of the spectrum of all $2^{n}-1$ principal submatrices of $G^{+}(0)$ for each of the $n$ ! possible pairing choices. This is prohibitive except when $n$ is relatively small. Condition 2) is relatively weak, while necessary for DIC it is not even sufficient for IC. On the other hand, property 2) of Lemma 2 provides that by evaluating the relative gain array for the $2^{n}-(n+1)$ principal submatrices of order $2, \cdots, n^{13}$ we can check all of the $n$ ! possible pairings; i.e., as rows of $G(s)$ are interchanged to study alternative pairings, the only change in the RGA is an interchange of the corresponding rows. The principal advantage of Condition 3 ) is that it only involves a test on $G^{+}(0)$ and not its principal submatrices.

\section{APPENDIX A NOTATION}

$\lambda_{\max }(A)$ The eigenvalue of $A$ which is largest in magnitude.

$\Lambda(A) \quad$ The Relative Gain Array of the matrix $A$.

$\mu(A) \quad$ The measure of the matrix $A$.

$\rho(A) \quad$ The spectral radius of the matrix $A$.

$\sigma(A) \quad$ The (eigenvalue) spectrum of the matrix $A$.

$a_{i j} \quad$ The $i j$ th element of the matrix $A$.

$A^{i j} \quad$ The $i j$ th cofactor of the matrix $A$.

$A^{+} \quad$ The matrix $A$ scaled to make its diagonal elements positive.

$A_{m} \quad$ The principal submatrix of $A$ consisting of rows and columns of $A$ indexed by $m$.

$\mathcal{C}_{+} \quad$ The open right-half plane.

$\overline{\mathcal{C}}_{+} \quad$ The closed right-half plane.

$\overline{\mathcal{C}}_{+}-\{0\}$ The closed right-half plane less the origin.

$D L \quad$ The set of all $D$-stable matrices.

$m_{r} \quad$ An index set of dimension $r$.

$\mathcal{P} \quad$ The set of all $\mathcal{P}$ matrices.

$S_{A} \quad$ The diagonal sign matrix derived from $A$.

$S(A) \quad$ The symmetric component of the matrix $A$.

$|A| \quad$ The matrix formed by taking the absolute values of the elements of $A$.

$\|A\|_{p} \quad$ The matrix norm of $A$ induced by the Holder $p$-norm on $\mathcal{R}^{n}$.

\section{ACKNOWLEDGMENT}

This work was performed while the first author was an Alexander von Humboldt Research Fellow at the Technical University of Munich and would not have been possible without the financial support of the Alexander von Humboldt

\footnotetext{
${ }^{13}$ The $n$ RGAs of order 1 are trivially positive.
} 
Stiftung. The authors wish to thank Richard D. Braatz for helpful criticisms and suggestions regarding this work.

\section{REFERENCES}

[1] E. H. Abed, “Strong $D$-stability," Syst. Contr. Lett., vol. 7, pp. 207-212, 1986.

[2] K. J. Arrow and M. McManus, "A note on dynamic stability," Econometrica, vol. 26, pp. 448-454, 1958.

[3] C. S. Ballantine, "Stabilization by a diagonal matrix," in Proc. Amer. Math. Soc., 1970, vol. 25, pp. 728-734.

[4] G. P. Barker, A. Berman, and R. J. Plemmons, "Positive diagonal solutions to the Lyapunov equations," Linear and Multilinear Algebra, vol. 5, pp. 249-256, 1976.

[5] E. H. Bristol, "On a new measure of interaction for multivariable process control," IEEE Trans. Automat. Contr., vol. AC-11, pp. 133-134, Jan. 1966.

[6] T. N. Chang and E. J. Davison, "Steady-state interaction indices for decentralized unknown systems," in Proc. 1986 Conf. Decision Contr., 1986, pp. 881-887.

[7] M. S. Chiu and Y. Arkun, "Decentralized control structure selection based on integrity considerations," Indust. Eng. Chem. Research, vol. 29 , pp. $369-373,1990$.

[8] E. J. Davison, "The robust decentralized control of a general servomechanism problem," IEEE Trans. Automat. Contr., vol. AC-21, no. 1, pp. 14-24, 1976

[9] C. A. Desoer and H. Haneda, "The measure of a matrix as a tool to analyze computer algorithms for circuit analysis," IEEE Trans. Circ. Theory, vol. 19, pp. 480-486, 1972.

[10] C. A. Desoer and M. Vidyasagar, Feedback Systems: Input-Output Properties. New York: Academic, 1975

[11] J. C. Doyle, "Analysis of feedback systems with structured uncertainties," in IEE Proceedings Part D, vol. 129, 1982, pp. 242-250.

[12] M. K. H. Fan and A. L. Tits, "Characterization and efficient computation of the structured singular value," IEEE Trans. Automat. Contr., vol. 31, pp. $734-743,1986$

[13] M. E. Fischer and A. T. Fuller, "On the stabilization of matrices and the convergence of linear iterative processes," in Proc. Cambridge Philosop. Soc., vol. 54, pp. 417-425, 1958.

[14] B. A. Francis, A Course in $H_{\infty}$ Control Theory. Berlin: SpringerVerlag, 1987

[15] M. Fujita, and E. Shimemura, "Integrity against arbitrary feedback loop failure in linear multivariable control systems," Automatica, vol. 26, no. 6, pp. 765-772, 1988.

[16] P. Grosdidier and M. Morari, "Closed-loop properties from steady-state gain information," Indust. Eng. Chem. Fund., vol. 24, no. 2, pp. 221-235, May 1985.

[17] P. Grosdidier and M. Morari, "Interaction measures for systems under decentralized control, Automatica, vol. 22, no. 3, pp. 309-319, May 1986.

[18] G. Guardabassi and A. Locatelli, "On the initialization problem in the parameter optimization of structurally constrained industrial regulators," Large Scale Syst., vol. 3, pp. 267-277, 1982.

[19] D. J. Hartfiel, "Concerning the interior of the $D$-stable matrices," Linear Algebra Appl., vol. 30, pp. 201-207, 1980.

[20] C. R. Johnson, "Sufficient conditions for D-stability," J. Econ. Theory, vol. 9, pp. 53-62, 1974.

[21] H. K. Khalil, "On the existence of positive diagonal $P$ such that $P A+A^{T} P<0$," IEEE Trans. Automat. Contr., vol. AC-27, pp. $181-184,1982$

[22] H. K. Khalil and P. V. Kokotovic, "D-stability and multiparameter singular perturbations," SIAM J. Contr. Optim., vol. 17, pp. 56-65, 1979.

[23] D. K. Le, O. D. I. Nwokah, and A. E. Frazho, "Multivariable decentralized integral controllability," Int. J. Contr., vol. 54, no. 2, pp. 481-496, 1991.

[24] V. Manousiouthakis, R. Savage, and Y. Arkun, "Synthesis of decentralized control structures using the concept of block relative gain," AIChE J., vol. 32, pp. 991-1003, June 1986.
[25] G. Mijares, J. D. Cole, N. W. Naugle, H. A. Preisig, and C. D. Holland, "A new criterion for the pairing of control and manipulated variables," AIChE J., vol. 32, pp. 1439-1449, 1986.

[26] M. Morari, "Robust stability of systems with integral control," IEEE Trans. Automat. Contr., vol. AC-30, pp. 574-577, June 1985.

[27] M. Morari and E. Zafiriou, Robust Process Control. Englewood Cliffs, NJ: Prentice-Hall, 1989.

[28] C. N. Nett and H. A. Spang, "Control structure analysis and synthesis: A missing link in the evolution of modern control theories," in Proc. Amer. Contr. Conf., Minneapolis, MN, June 1987.

[29] A. Niederlinski, "A heuristic approach to the design of linear multivariable interacting control systems," Automatica, vol. 7, pp. 691-701, 1971.

[30] O. D. I. Nwokah, A. E. Frazho, and D. K. Le, "A note on decentralized integral controllability," Int. J. Contr., vol. 34, pp. 485-494, 1993.

[31] D. H. Owens, Feedback and Multivariable Systems. London: Peter Peregrinus Ltd., 1978.

[32] A. K. Packard, "What's New With $\mu$ : Structured Uncertainty in Multivariable Control," Ph.D. dissertation, University of California, Berkeley, 1988.

[33] S. Skogestad and M. Moarai, "Variable selection for decentralized control," presented at AIChE Annual Meeting, Washington, Nov. 1988.

[34] C. C. Yu and M. K. Fan, "Decentralized integral controllability and $D$-stability," Chem. Eng. Sci., vol. 45, no. 11, pp. 3299-3309, 1990.

[35] C. C. Yu and W. L. Luyben, "Design of multiloop SISO controllers in multivariable processes," Indus. Eng. Chem. Process Design and Devel., vol. 15, pp. 498-503, 1986.

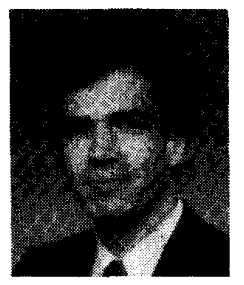

Peter Campo received the B.S. degree in chemical engineering from Rice University, Houston, TX, in 1984, and the Ph.D. degree in chemical engineering from the California Institute of Technology, Pasadena, in 1989

In 1989 and 1990 he spent 14 months at the Technical University of Munich as an Alexander von Humboldt Fellow pursuing research interests in decentralized control and in the design and analysis of controller implementations providing Antiwindup and Bumpless transfer operation in the face of input saturation nonlinearities. Since 1990 he has been a Control Systems Engineer in the Control System and Electronic Technologies Laboratory of the General Electric Corporate Research and Development Center, Schenectady, New York.

Dr. Campo's current research interests include applications of linear and nonlinear control theory and development of machine vision systems for feedback control of manufacturing processes.

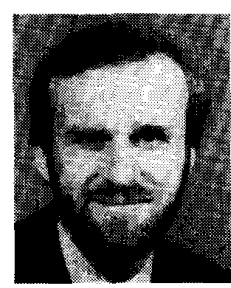

Manfred Morari (M'87) obtained the diploma from ETH Zürich and the Ph.D. from the University of Minnesota, both in chemical engineering.

Dr. Morari's interests are in the areas of process control and design. He is the McCollum-Corcoran Professor and Executive Officer for Control and Dynamical Systems at the California Institute of Technology, Pasadena. He has held appointments with the University of Wisconsin, Exxon R\&E and ICI and has consulted internationally for a number of major corporations. In recognition of his research contributions, he received numerous awards, among them the Donald P. Eckman Award of the Automatic Control Council. He was recently elected to the National Academy of Engineering. 